\title{
Minilaparoscopic Resection of Mesenteric Cystic Lymphangioma in an Infant
}

\author{
Arpit Amin, MD, Nishank Nooli, MD , Samir Pandya, MD \\ Department of Surgery, New York Medical College, Westchester Medical Center, Valhalla, New York (all authors).
}

\begin{abstract}
Introduction: Abdominal mesenteric cystic lymphangiomas are congenital lymphatic malformations found most commonly within the small bowel mesentery, omentum, and mesocolon. These lesions may be detected incidentally on imaging studies or may present with bowel obstruction. Treatment of choice is surgical enucleation with possible segmental intestinal resection.

Case Description: This report describes the use of minilaparoscopy in the treatment of abdominal mesenteric cystic lymphangioma (MCL) in a child, along with a review of the current literature on laparoscopic treatment. Our patient, who had a prenatal diagnosis of an intra-abdominal mass, had developed postprandial emesis. Imaging work-up with ultrasonography and CT scan revealed an intra-abdominal mass suspicious for lipomatous tumor. The patient underwent minilaparoscopy-assisted resection of the intra-abdominal mass, along with the resection of small bowel that was adherent to the tumor. The final pathology showed that the mass was an MCL.
\end{abstract}

Discussion: The traditional treatment for MCL has been open surgical resection. Recently, laparoscopic surgical excision of mesenteric cysts in children has been reported in the literature.

Key Words: Abdominal mesenteric cystic lymphangioma, Minilaparoscopy

Citation Amin A, Nooli N, Pandya S. Minilaparoscopic resection of mesenteric cystic lymphangioma in an infant. CRSLS e2015.00083. DOI: 10.4293/CRSLS.2015.00083.

Copyright $\odot 2015$ by SLS, Society of Laparoendoscopic Surgeons. This is an open-access article distributed under the terms of the Creative Commons Attribution-Noncommercial-ShareAlike 3.0 Unported license, which permits unrestricted noncommercial use, distribution, and reproduction in any medium, provided the original author and source are credited.

Address correspondence to: Arpit Amin, MD, Department of Surgery, New York Medical College, Westchester Medical Center, 100 Woods Road, Taylor Pavilion E173, Valhalla, NY 10595 USA. Telephone: 973-885-2591, Fax: (914) 493-1679, E-mail: arpitamin@gmail.com

\section{INTRODUCTION}

Lymphangioma is a congenital malformation of the lymphatic system. It can occur throughout the body. ${ }^{1}$ Its most common location is in the head and neck region, but it may develop intra-abdominally. ${ }^{1}$

Abdominal cystic lymphangioma is usually found in the small-bowel mesentery, followed by the omentum and mesocolon. ${ }^{2}$ Its clinical presentation is highly variable, ranging from asymptomatic, identified incidentally, to overt, in the form of bowel obstruction or peritonitis. ${ }^{3}$ The diagnosis can be established with the help of abdominal ultrasonography, computed tomography (CT), or laparoscopy. ${ }^{4}$ Treatment for abdominal cystic lymphangioma is surgical enucleation, with the possibility of segmental intestinal resection when it is intimately adherent to the intestine. ${ }^{2}$ The approach has most often been open surgery through a laparotomy incision.
We describe the use of minilaparoscopy in the management of abdominal mesenteric cystic lymphangioma (MCL) in a 1-month-old girl, along with a review of the current literature on the laparoscopic treatment of abdominal MCL.

\section{CASE DESCRIPTION}

\section{Preoperative Evaluation}

A 1-month-old girl who had postprandial emesis was referred for evaluation for a mesenteric mass detected by CT. It had been found before birth by obstetric ultrasonography and was presumed to be cystic. The adnexa were reportedly normal. No masses were palpable on rectal examination. The differential diagnoses included a type IV sacrococcygeal teratoma. However, the mass did not appear to arise from the coccyx, 


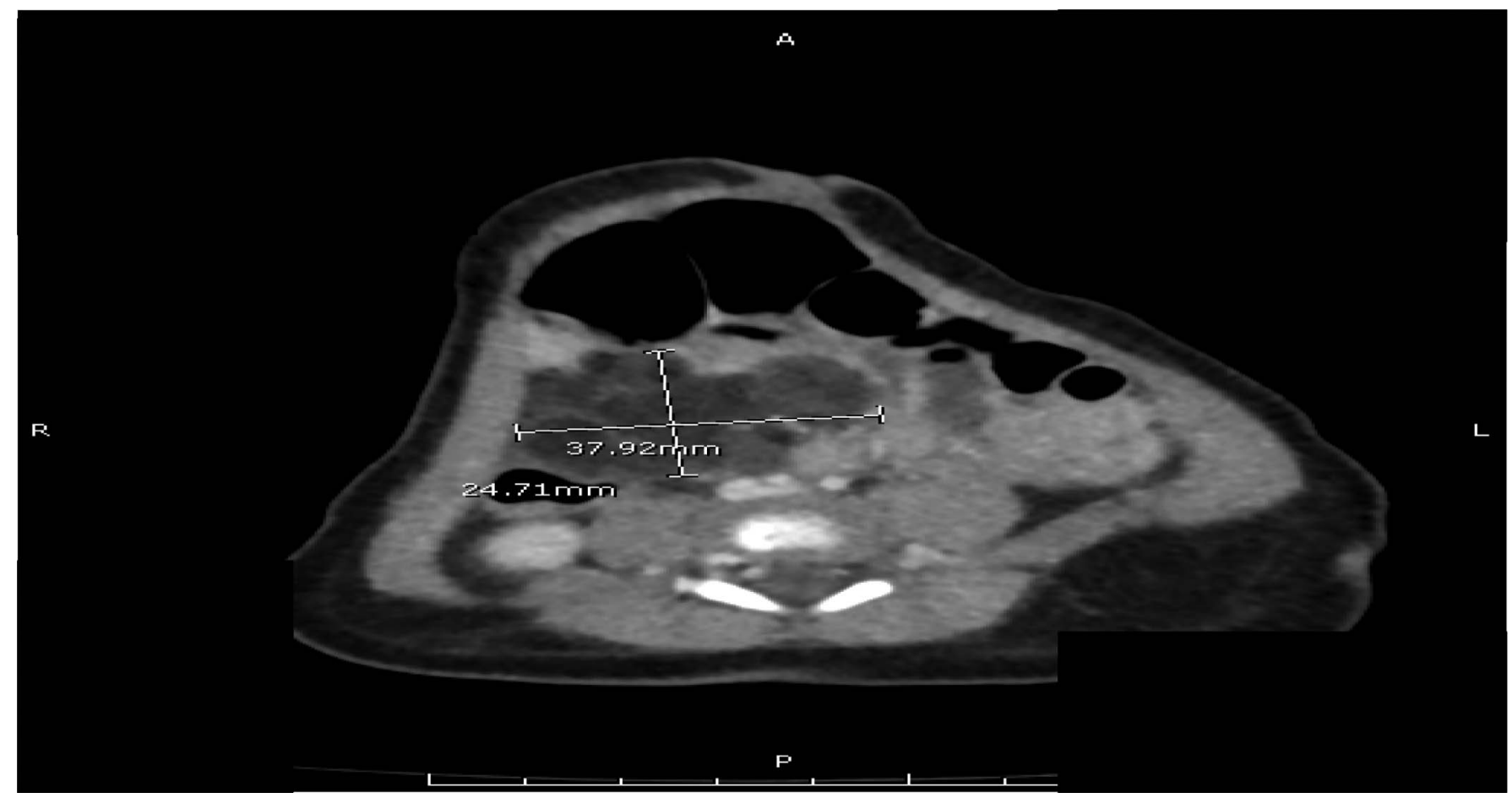

Figure 1. Axial view of an abdominal CT scan showing a $2.5 \times 3.8 \times 5.3$-cm lobulated mass to the right of the midline below the liver, mainly of fat density, showing thin enhancing septa traversed by mesenteric vessels, with no calcification, ossification, fluid levels, or evidence of bowel obstruction.

making this diagnosis unlikely. After birth, the patient underwent a CT scan of the abdomen and pelvis with contrast that revealed a $2.5 \times 3.8 \times 5.3$-cm lobulated mesenteric mass suspicious for mesenteric lipoblastoma. (Figure 1).

A laparoscopic evaluation was planned for more accurate diagnosis and potential definitive management. Given the young age and small size of the patient, we elected to use minilaparoscopic instruments.

\section{Operative Findings}

Pneumoperitoneum was obtained by placing a 3-mm trocar in the umbilical region. Copious chylous fluid was noted in the pelvis and in the right lower quadrant. The mass was found to be in the left upper quadrant, arising from the mesenteric border of a proximal jejunal loop extending down to the root of the mesentery. Two 3 -mm ports were placed in the right hemiabdomen. The mass was noted not to involve any major mesenteric vessels and was sufficiently mobile that we could deliver the mass and involved bowel directly through the umbilicus. We extended the umbilical port incision cephalad, delivered the mass through the midline, and resected the mass en bloc with $\sim 20 \mathrm{~cm}$ of small bowel. A primary end-to-end hand-sewn anastomosis was performed, and the mesenteric defect was closed. All the chylous fluid was removed by suctioning. The fascia at the midline incision was closed. The skin at the midline abdominal incision and all port sites were approximated with absorbable sutures and skin adhesive (Figure 2).

\section{Postoperative Course}

Pathology revealed the mesenteric mass to be an MCL (Figures 3 and 4). The resection margins were negative. The patient was discharged on postoperative day 7 after resumption of bowel function. The postoperative course was complicated by a superficial surgical site infection that was treated with antibiotics. The infant's symptoms resolved after surgery, and she was tolerating her diet well on follow-ups at 1 month and 1 year.

\section{DISCUSSION}

Abdominal MCL is a rare, benign malformation of the lymphatic system. It has been postulated that the mal- 


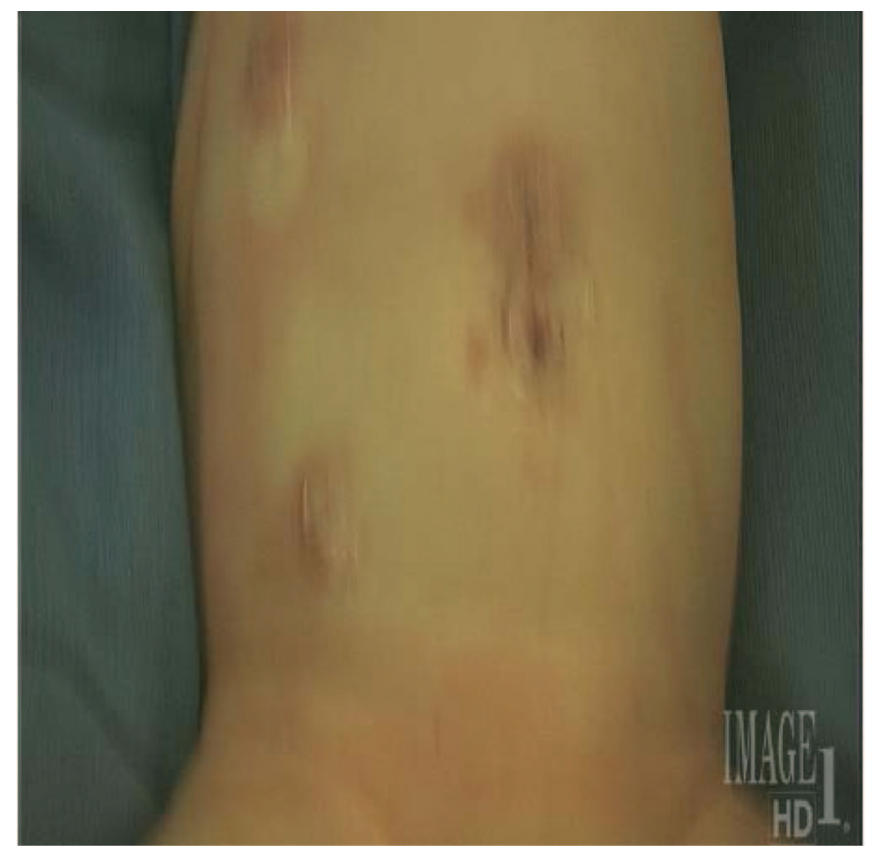

Figure 2. Photograph obtained immediately after surgery, demonstrating the cosmetic appearance of all the minilaparoscopic port sites and the midline abdominal incision.

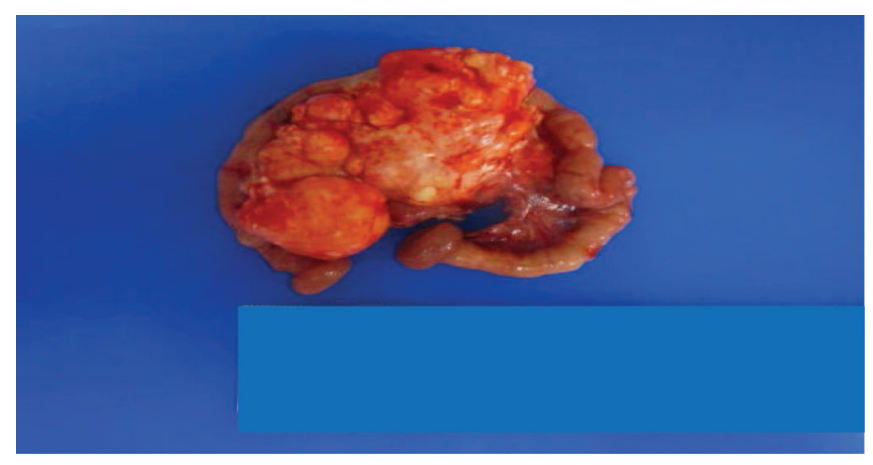

Figure 3. Gross view showing small bowel attached to a yellowwhite, multinodular, soft mass with thin-walled cysts of various sizes.

formation is caused by the defective connection of the fetal lymphatic sacs with the venous system.5,6 Abdominal MCL is usually found in infants and children. ${ }^{2}$ Most cases are diagnosed within the first 5 years of life. ${ }^{4}$

The clinical presentation of abdominal MCL is highly variable. As described in our case, it may be diagnosed incidentally on imaging studies performed antenatally. It also may cause small-bowel obstruction and present acutely with abdominal pain, vomiting, and fever. In some cases, it presents as a palpable abdominal mass. ${ }^{3}$

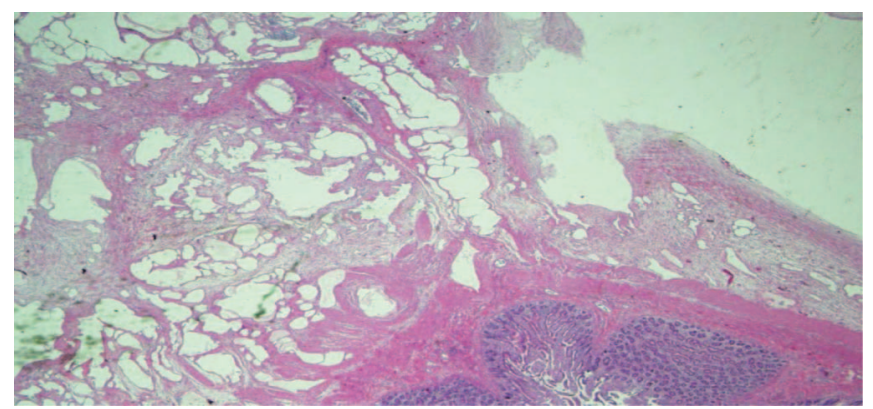

Figure 4. Microscopic examination of mesenteric mass revealed multiple small and large thin- to thick-walled lymphatic channels in the small intestine mesentery.

Infrequently, the cystic lymphangioma ruptures, causing peritonitis. ${ }^{2}$

Differential diagnosis of abdominal MCL includes a benign abdominal cyst (pseudocyst, dermoid cyst, enteric duplication cyst, or lymphocele) and neoplastic lesion (lipoma, teratoma, leiomyosarcoma, or liposarcoma). ${ }^{2}$ Losanoff et $\mathrm{al}^{7}$ have recently proposed classification of abdominal MCL into 4 categories: type I is pedicled, type II is sessile, type III has a retroperitoneal extension, and type IV is multicentric. Type I MCL can be resected without small-bowel resection, type II usually requires smallbowel resection, ${ }^{7}$ and types III and IV may not be completely resectable. ${ }^{7}$ Based on this classification, the patient in our case had a type II MCL.

Plain abdominal radiography may reveal small-bowel loop displacement with an intra-abdominal soft tissue mass. ${ }^{7}$ On ultrasonography, abdominal MCL usually presents as a hypoechogenic multilocular mass. ${ }^{3}$ A CT scan usually reveals a cystic mass with homogeneous fluid attenuation, with density ranging from -4 to -34 Hounsfield units. ${ }^{2}$ Abdominal ultrasonography is the initial diagnostic modality of choice and is the recommended modality for long-term follow-up. ${ }^{7}$

Abdominal MCL histology is characterized by cystic spaces lined with a thin layer of endothelium and connective tissue. ${ }^{2}$ Small lymphoid aggregates within the cystic wall aid in the diagnosis of MCL. ${ }^{7}$ Chylous, serous, or serosanguineous fluid may be found within the abdominal MCL. ${ }^{4}$ Immunohistochemical analysis usually reveals lymphatic spaces lined with endothelial cells that are positive for CD31, D2-40, CD34, and factor VIII-related antigen and negative for calretinin. 4,8

The treatment of abdominal MCL is complete surgical excision. ${ }^{2}$ The mass tends to recur and invade neighboring structures. ${ }^{7}$ Historically, laparotomy with surgical ex- 
Minilaparoscopic Resection of Mesenteric Cystic Lymphangioma in an Infant, Amin A et al.

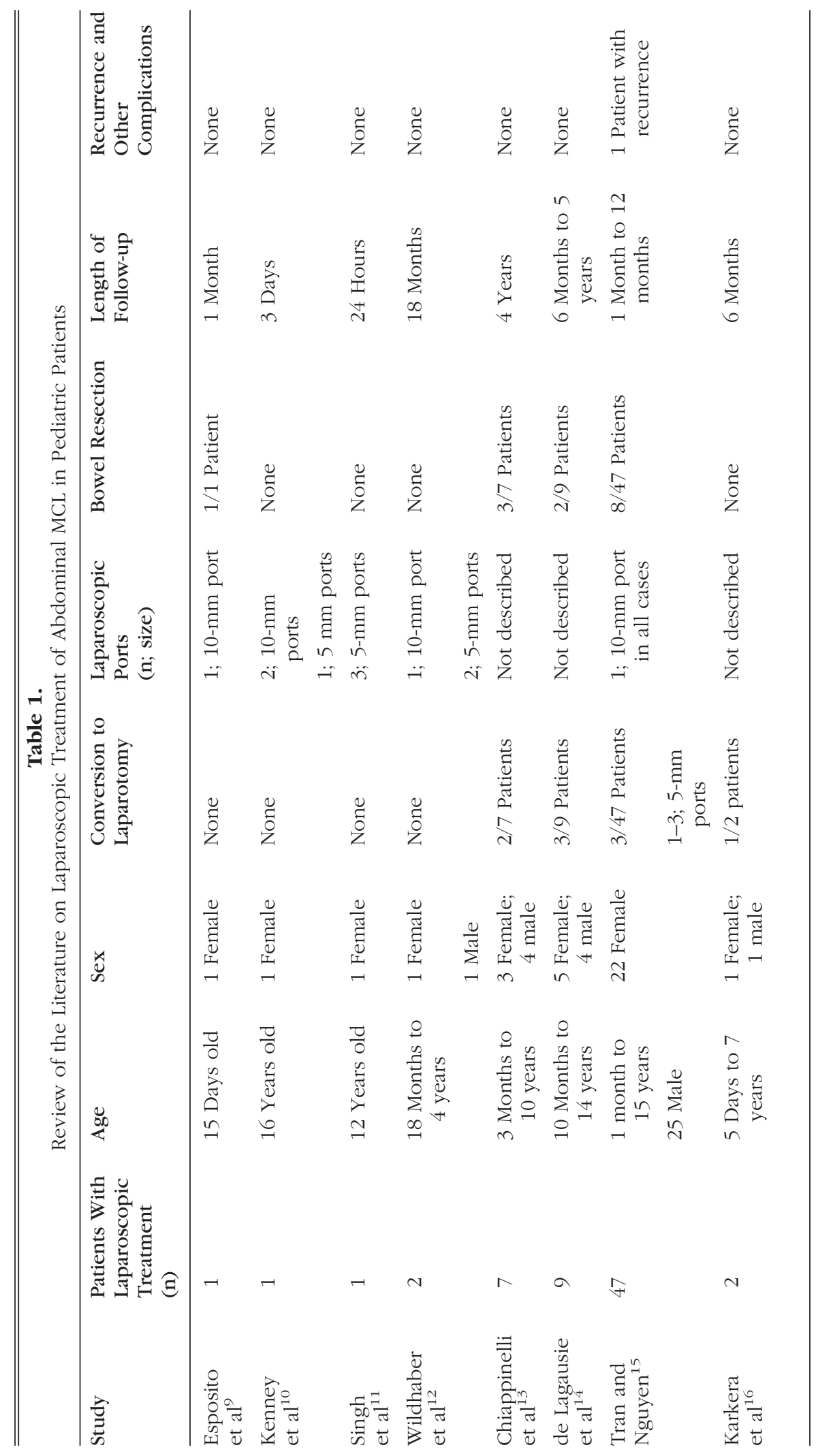


cision of abdominal MCL has been performed. More recently, laparoscopic surgical excision has been reported in children. ${ }^{9}$

We performed a literature search to review laparoscopic management of abdominal or retroperitoneal MCLs in pediatric patients reported within the past 20 years (Table 1). ${ }^{9-16}$ The first case report of laparoscopic treatment of pediatric abdominal MCLs was reported in 1996 by Kenney and colleagues ${ }^{10}$ in a 16-year-old girl. The largest case series, comprising 47 pediatric patients with abdominal MCL treated with laparoscopy, was reported in 2010 by Tran and Nguyen. ${ }^{15}$ The youngest patient reported in the literature to have undergone laparoscopic treatment of an abdominal MCL was a 15-day-old girl.9 The total number of ports in the laparoscopic treatment of abdominal MCL ranges from 1 to $4 .^{9-16}$ The smallest port size is $5 \mathrm{~mm}$, and the largest is $10 \mathrm{~mm} \cdot{ }^{9-16}$

Of note, we used 3-mm minilaparoscopic ports to perform mobilization and dissection of the abdominal MCL in our case. Adjunctive laparoscopic techniques, such as cyst aspiration to decrease the lesion size and use of an EndoCatch bag (Covidien, Minneapolis, Minnesota) to prevent intra-abdominal spillage of cyst content during retrieval, have been reported. ${ }^{9-16}$ All laparoscopic cases requiring bowel resection were performed extracorporeally. The longest reported follow-up after laparoscopic treatment of abdominal MCL is 5 years. ${ }^{14}$ One patient was reported to have a recurrence at the 3-month follow-up. ${ }^{15}$

Advantages of the minimally invasive approach include less pain, less pulmonary morbidity, and lower risk of postoperative adhesions. ${ }^{9}$ Compared to traditional laparoscopic instruments, minilaparoscopic instruments occupy less space because of their thinner profile and thereby allow for a better view in the amplified visual field provided by a laparoscopic camera. ${ }^{17}$ The operative space is restricted in pediatric patients because of their small size. In the pediatric population, minilaparoscopy provides a dual advantage of occupying less space in a small operative field without compromising on the maneuverability of surgical instruments, while offering cosmesis superior to that of conventional laparoscopy. The minilaparoscopic approach may be used to evaluate the relationship of the abdominal MCL with surrounding structures to assess whether small-bowel resection is necessary. In cases that do not requiring bowel resection, these lesions may be aspirated and removed via small incisions, thus avoiding the morbidity of a laparotomy incision. In cases necessi- tating bowel resection, a small minilaparoscopy-assisted limited incision may be sufficient to perform extracorporeal bowel resection and anastomosis. For surgically nonresectable abdominal MCL, intracystic injection of the sclerosing agent OK432 has been demonstrated to be effective. ${ }^{7}$

In conclusion, our case report demonstrates that minilaparoscopy can be applied in the treatment of abdominal MCL in children, with an excellent cosmetic outcome.

\section{References:}

1. Okazaki T, Iwatani S, Yanai T, et al. Treatment of lymphangioma in children: our experience of 128 cases. J Pediatr Surg. 2007;42:386-389.

2. Mendez-Gallart R, Solar-Boga A, Gomez-Tellado M, SomozaArgibay. Giant mesenteric cystic lymphangiomas in an infant presenting with acute bowel obstruction. Can J Surg. 2009;52: E42-E43.

3. Steyaert H, Guitard J, Moscovici J, Juricic M, Vaysse P, Juskiewenski S. Abdominal cystic lymphangiomas in children: benign lesions that can have a proliferative course. J Pediatr Surg. 1996;31:677-680.

4. Rami M, Mahmoudi A, El Madi A, et al. Giant cystic lymphangiomas of the mesentery: varied clinical presentation of 3 cases. Pan Afr Med J. 2012;12:7.

5. Galifer RB, Pous JG, Juskiewenski S, et al. Intra-abdominal cystic lymphangiomas in childhood. Prog Pediatr Surg. 1978;11: 173-238.

6. Godart S. Embryological significance of lymphangioma. Arch Dis Child. 1966;41:204-206.

7. Losanoff JE, Richman BW, El-Sherif A, et al. Mesenteric cystic lymphangioma. J Am Coll Surg. 2003;196:598-603.

8. Suthiwartnarueput W, Kiatipunsodsai S, Kwankua A, Chaumrattanakul U. Lymphangioma of the small bowel mesentery: a case report and review of the literature. World J Gastroenterol 2012;18:6328-6332.

9. Esposito C, Alicchio F, Savanelli A, et al. One-trocar ileocolic resection in a newborn infant with a cystic lymphangioma of the small-bowel mesentery. J Laparoendosc Adv Surg Tech A. 2009;19:447-449.

10. Kenney B, Smith B, Bensoussan AL, et al. Laparoscopic excision of a cystic lymphangioma. J Laparoendosc Surg. 1996; 6:S99-S101.

11. Singh RR, Govindarajan KK, Bowen C, et al. Retroperitoneal cystic lymphangioma: a rare presentation in childhood, treated laparoscopically. J Laparoendosc Adv Surg Tech A. 2009;19:249-250. 
Minilaparoscopic Resection of Mesenteric Cystic Lymphangioma in an Infant, Amin A et al.

12. Wildhaber BE, Chardot C, Le Coultre C, et al. Total laparoscopic excision of retroperitoneal cystic lymphangioma. J Laparoendosc Adv Surg Tech A. 2006;16:530-533.

13. Chiappinelli A, Forgues D, Galifer RB, et al. Congenital abdominal cystic lymphangiomas: what is the correct management? J Matern Fetal Neonatal Med. 2012;25:915-919.

14. de Laguasie P, Bonnard A, Berrebi D, et al. Abdominal lymphangiomas in children: interest of the laparoscopic approach. Surg Endosc. 2007;21:1153-1157.
15. Tran NS, Nguyen TL. Laparoscopic management of abdominal lymphatic cyst in children. J Laparoendosc Adv Surg Tech A. 2012;22:505-507.

16. Karkera PJ, Sandlas GR, Ranjan RR, et al. Intra-abdominal cystic lymphangiomas in children: a case series. Arch International Surg. 2012;2:91-95.

17. Carvalho GL, Loureiro MP, Bonin EA. Renaissance of minilaparoscopy in the NOTES and single port era. JSLS. 2011;15: $585-588$. 